\title{
LAS MENORES EXTRANJERAS NO ACOMPAÑADAS EN MELILLA: ¿MENORES O EXTRANJERAS?
}

MARISA ESCÁMEZ PASTRANA

Diplomada en Educación Social

Diplomada en Trabajo Social

Máster Universitario Oficial en "Problemas Sociales" por la UNED

Este artículo es una versión breve deITRABAJO FIN DE MÁSTER OFICIAL EN PROBLEMAS SOCIALES, leído en Madrid el 28 de Septiembre de 2011 y valorado con Matrícula de Honor por el Tribunal calificador. Se trata de un trabajo previo al DOCTORADO EN ANÁLISIS DE LOS PROBLEMAS SOCIALES.

Como este trabajo habla de "ellas", las Menores Extranjeras No Acompañadas, nos referiremos a este asunto siempre en femenino, aunque en algunos casos pueda extenderse por supuesto, también a ellos.

A la hora de abordar un estudio en profundidad acerca de las menores extranjeras inmigrantes no acompañadas en Melilla, se hace necesario incidir en las diferencias que manifiesta este colectivo en nuestra ciudad con respecto a otras provincias españolas, además de los contrastes con respecto a los proyectos migratorios iniciados por los chicos procedentes de las mismas bolsas de inmigrantes que se encuentran en Marruecos ${ }^{1}$.

${ }^{1}$ En:http: / / www.uca.es/grupos-inv/migraciones/inmigrante_marroqui/volumen-ydistribucion/ poblacion-extranjera-de-origen-marroqui-en-andalucia/?searchterm= bolsas de inmigrantes.

En las últimas décadas del siglo XX los flujos de marroquíes hacia la península han tendido a intensificarse. En los años setenta los inmigrantes marroquíes presentan dos perfiles claramente diferenciados: una proporción importante de ellos eran judíos de clase media cuya motivación para asentarse en España respondía a factores políticos y religiosos; el resto lo constituían inmigrantes musulmanes obreros o trabajadores agrícolas. Los años ochenta marcaron el cambio decisivo hacia una migración de tipo económico (obreros, trabajadores agrícolas, trabajadoras del servicio doméstico), que crece rápidamente y que debe enfrentarse a los primeros esfuerzos de control de flujos migratorios por parte de los gobiernos españoles, dando origen al círculo vicioso de acumulación de bolsas de inmigrantes sin papeles marroquíes y regularizaciones extraordinarias. A partir del año 2000, se inicia el proyecto migratorio de cientos de jóvenes que aœen perdura. En Bernabé López García "La inmigración de magrebíes y africanos. Asumir la vecindad”. Alicia Alted y Almudena Asenjo (Coord.). De la España que emigra a la España que acoge. Caja Duero-Fundación Largo Caballero, Madrid, 2006, pp. 480-491. 
El o la MEINA (menor extranjera inmigrante no acompañada) en el Derecho comunitario se define como "el menor de dieciocho años que llega al territorio de los Estados miembros sin ir acompañado de un adulto responsable del mismo, ya sea legalmente o con arreglo a los usos y costumbres, y mientras no esté efectivamente bajo el cuidado de tal adulto responsable de él" ${ }^{2}$ (Directivas 2003/9/CE del Consejo, de 27 de enero de 2003 y la Directiva 2001/55/CE, del Consejo, de 20 de julio de 2001). En este concepto se incluye también "a los menores que dejan de estar acompañados después de haber entrado en el territorio de los Estados miembros".

De la misma manera que en el resto de los países de la Unión Europea, España está obligada por distintos tratados a la atención y acogida de menores extranjeras inmigrantes no acompañadas (MEINAS). La entrada irregular de estas menores se encuentra reglamentada en España a través de una combinación de las normativas de extranjería y de protección de menores en situación de riesgo o desamparo. Por este motivo, la normativa de extranjería establece que una vez que se comprueba su minoría de edad, la extranjera y menor no acompañada, debe ser puesta a disposición de los servicios de protección de menores de las Comunidades Autónomas puesto que estas son las Administraciones competentes para declarar su desamparo, asumir su tutela y ejercer las medidas de protección necesarias.

Una de las garantías con las que cuentan las menores extranjeras no acompañadas es la intervención del Ministerio Fiscal, que posee el mandato legal de velar por el cumplimiento adecuado de los procedimientos llevados a cabo con menores y por el ejercicio de sus derechos, por lo que debe conocer todas las decisiones que se tomen con MEINAS. En concreto, las Fiscales Delegadas de Extranjería tienen asignadas las funciones de intervenir coordinando, supervisando y trasladando las pautas a seguir en los expedientes sobre determinación de edad y repatriación de menores extranjeras no acompañadas ${ }^{3}$.

La mayoría de las menores llegan a España realizando peligrosas travesías, motivadas mayoritariamente por cuestiones económicas, ya que algunos y algunas en nuestro caso,

2 (Directivas 2003/9/CE del Consejo, de 27 de enero de 2003 y la Directiva 2001/55/CE, del Consejo, de 20 de julio de 2001). En este concepto se incluye también "a los menores que dejan de estar acompañados después de haber entrado en el territorio de los Estados miembros".

Ambas definiciones tienen su origen en la Resolución del Consejo de Europa 97/C 221/03, de 26 de junio 1997, relativa a los menores no acompañados nacionales de países terceros, aunque esta no incluía los menores que se quedan solos después de entrar en territorio de la Unión.

3 Secretaría de Estado de Inmigración y emigración. http://extranjeros.mtin.es/es/ $\underline{\text { RedEuropeaMigraciones }}$ 
manifiestan que vienen con el objetivo de conseguir "papeles" ${ }^{\text {y }}$ trabajo, para poder vivir en España y en menor medida, enviar dinero a sus familias:

-"Aquí estoy muy valorada, en Marruecos, nada. Yo quiero tener poder y por eso también quiero ser policía" Entrevista a Menor.III ${ }^{5}$ Las entrevistas realizadas a las informantes clave, menores o funcionarios/as, han sido recogidas y numeradas, y serán referenciadas a lo largo del trabajo según pertinencia.

Las entrevistas a MENAS (E.M.I a X); entrevista a Ex-MENAS (E.E.M I); entrevista a DIRECTORA GENERAL del Menor y la Familia (E.D.G.M); entrevista a FUNCIONARIOS...;

-"Quiero estudiar, si Dios quiere, terminar mi PCPI de comercio y formar una familia propia, porque no sé nada de la mía, aunque mi madre sabe que estoy aquí y que me tratan bien”. E.M.IV;

-"Quiero trabajar y ganar dinero para vivir en España pero para mí no para nadie de Marruecos. No quiero volver, me gustaría aprender peluquería porque yo quiero quedarme aquí. Me gusta bailar y puedo ir a "Fama” (Programa de Televisión)"E.M.V;

- "Tengo muchas ganas de cumplir mayoría de edad para vivir y trabajar aqui”. E.M.VIII;

- "Quiero seguir estudiando y ser peluquera porque trabajan y ganan mucho”. EM.X.

La decisión de emigrar puede ser personal o familiar, constatándose la influencia que tiene el "mito europeo" a la hora de la disponibilidad para iniciar el proyecto migratorio, sin olvidar que en el trasfondo de estas migraciones se encuentra la precaria situación económica y social de estas menores en sus países.

- "La tomó mi madre. Me dejó aquí porque se lo dijeron en el hospital” E.M.I.; "La tomó mi madre con mi primo" E.M.VII; "La tomó mi madre. Me trajo ella. Ella es la que sabía cómo era esto”. E.M.IX; "Yo no decidí nada, lo decidió mi hermano"E.M.X;"Mi tía me trajo porque yo estaba muy mal”. E.M.XI; "Mi madre me dijo que me tenía que venir a Melilla, que aquí estaría bien y no se ha equivocado”. E.E.M.

-"La tomé yo. Me escapé". E.M.II y E.M.VI; "Yo, después que me lo dijera mi hermano y mi madre" E.M.III.

-"La decisión la toma por mí una mujer que se ofrece para ayudarme"E.M.IV; "Yo he venido porque me ha invitado una mujer que trabaja en Melilla"E.M.V; "Lo pensó mi hermana y una mujer que trae a niñas” E.M.VIII.

${ }^{4}$ Es la forma habitual entre ellas de referirse a la documentación. "Tener papeles" es estar documentada.

${ }^{5}$ Las entrevistas realizadas a las informantes clave, menores o funcionarios/as, han sido recogidas y numeradas, y serán referenciadas a lo largo del trabajo segœn pertinencia.

Las entrevistas a MENAS (E.M.I a X); entrevista a Ex-MENAS (E.E.M I); entrevista a DIRECTORA GENERAL del Menor y la Familia (E.D.G.M); entrevista a FUNCIONARIOSÉ 
Un dato relevante según Jiménez ${ }^{6}$ es que el 70\% de los jóvenes marroquíes quieren emigrar.

La intervención que se realiza en España con estas menores está recogida en el Protocolo de Menores Extranjeros No Acompañados, aprobado el 14 de noviembre de 2005 por el grupo de trabajo del Observatorio de la Infancia, que asigna las funciones de las Administraciones Públicas y Organismos que intervienen en esta materia y está redactado conforme al artículo 92 del Reglamento de Extranjería, artículo que desarrolla los procedimientos que deben llevarse a cabo con las menores extranjeras no acompañadas localizadas en España. En nuestro país, todas las MEINAS tienen garantizado el derecho a la educación y todas las que se hallen en situación de riesgo o bajo la tutela o guarda de una Entidad de Protección de Menores, incluso cuando no estén autorizados a residir, tienen derecho a la asistencia sanitaria y a los demás servicios públicos ${ }^{7}$.

Hace más de veinte años que se localizaron en España las primeras MEINAS y su número ha ido en aumento año tras año, hasta el punto de saturar los centros de primera acogida de los servicios de protección de menores de las Ciudades y Comunidades Autónomas, sobre todo los centros situados en los territorios de la frontera sur como es el caso de Andalucía, Canarias, Ceuta y Melilla y los centros situados en las grandes ciudades, como el caso de Madrid y Barcelona. Por este motivo, se creó un Programa Especial ${ }^{8}$ de traslado y atención de MEINAS o desplazadas, recibiendo el apoyo económico de la Administración General del Estado ${ }^{9}$ las Ciudades Autónomas de Ceuta, Melilla y la Comunidad Autónoma de Canarias.

Con este apoyo y debido a la necesidad de transformar instalaciones e infraestructura y dotar a edificios ya construidos o en desuso como nuevos Centros de acogida gestionados por la Ciudad Autónoma por el gran número de menores que han accedido a la ciudad como MEINAS y su situación; la Ciudad Autónoma ha acometido la rehabilitación y ampliación del Centro Educativo Residencial de Menores "Fuerte de la Purísima

\footnotetext{
${ }^{6}$ Manuel Jiménez Álvarez. Los niños de la calle y el fenómeno de la emigración de menores: análisis de los contextos migratorios en el área urbana de Tánger. Agencia Española de Cooperación, Madrid, 2001.

${ }^{7}$ Reglamento aprobado por el Real Decreto 2393/2004, de 30 de diciembre, que desarrolla Ley Orgánica 4/2000, de 11 de enero, sobre derechos y libertades de los extranjeros en España y su integración social, modificada por las Leyes Orgánicas 8/2000, de 22 de diciembre, 11/2003, de 29 de septiembre y 4/2003, de 20 de noviembre.

${ }^{8}$ Resolución de 24 de marzo de 2009 (BOE de 2-4-2009). Fondos de Apoyo a la acogida y la integración de inmigrantes así como el refuerzo educativo de los mismos.

${ }^{9}$ Informe producido por la Red Europea de Migraciones, elaborado por el Punto de Contacto Nacional de España "La politica de acogida, repatriación y acuerdos para la integración de los Menores extranjeros no acompañados". Madrid. Junio 2009.
} 
Concepción” (CEMP), “Casa Hogar Nuevo Futuro”, Pabellón de niños, niñas y Centro Hermano "Eladio Alonso" del Centro Asistencial, Casa de las Esclavas de la Inmaculada Niña (Divina Infantita)... ${ }^{10}$.

Así, se considera que las MEINAS, llamados MENAS en Melilla, atendidos por los servicios de protección a la infancia pueden llegar a suponer hasta el cincuenta por ciento de las menores en acogimiento algunas regiones ${ }^{11}$. En Melilla, según los datos de la Dirección General del Menor y la Familia de la Consejería de Bienestar Social y Sanidad de la Ciudad Autónoma, los y las MENAS superan el ochenta y cinco por ciento las Menores atendidos por los Servicios de protección a la infancia. La alta afluencia de estos menores en situación de desamparo, tanto de sexo masculino como femenino crea situaciones de alarma social en todo el país ${ }^{12}$.

En cuanto a las poblaciones de los centros, el número de menores de sexo masculino acogidos es vacilante y oscila con mucha frecuencia. Fundamentalmente se trata de menores de origen marroquí ${ }^{13}$. Estos MENAS masculinos suelen alternar estancias en los diferentes centros de la ciudad que les son asignados, con estancias de tiempo más o menos cortas en su país de origen, para volver a ingresar nuevamente en los centros. Esto sucede porque cada vez que un menor es interceptado en algún punto de la ciudad por las Fuerzas y Cuerpos de Seguridad del Estado, se inicia de nuevo el procedimiento establecido.

En el caso que nos ocupa, las niñas menores extranjeras no acompañadas, su invisibilidad, ocultación y a veces nuestra indiferencia, impide en ocasiones que sean percibidas como sujetos con derechos, en este caso especiales por su condición de menores de edad ${ }^{14}$. Por otra parte se está haciendo evidente un hecho relevante: la feminización de los flujos migratorios y políticas europeas de inmigración ${ }^{15}$.

\footnotetext{
${ }^{10}$ Fuente: Dirección General del Menor y la Familia de la Consejería de Bienestar Social y Sanidad de la Ciudad Autónoma de Melilla.

${ }^{11} \mathrm{http}$ / / www.dianova.es/content/que-hacemos-menas-residencial.html

12 "La difusión desproporcionada de conflictos puntuales está generando el rechazo en el entorno vecinal de los centros de acogida, terror en las escuelas que los acogen y da lugar a enfrentamientos absurdos". SOS Racismo. http://medios.mugak.eu/noticias/noticia/96151

${ }^{13}$ Véase "el perfil de la mayor parte de estos menores es el de joven marroquí entre 15 y 18 años que viene a España a buscarse la vida, en Isabel Lázaro González. Prolegómenos: Derechos y Valores. "Menores extranjeros no acompañados. La situación en España”. Universidad Militar Nueva Granada. Bogotá. Colombia. Enero/junio 2007, pp.149-162.

${ }^{14}$ http://www.ujaen.es/investiga/ideo/jornadas/recursos/actasConISBN.pdf http://menoressolos.blogspot.com/2009/06/las-ninas-y-adolescentes-que-emigran.html

${ }^{15}$ Una de las características de los movimientos migratorios a finales del siglo XX y principios del siglo XXI es que la iniciativa de emigrar e instalarse en otro país la han tomado progresivamente las mujeres por la
} 
Además se está haciendo notar una nueva realidad: la "feminización de la pobreza" que no es de las mujeres o niñas en general, sino de las mujeres o niñas en específicas situaciones de vulnerabilidad adicional, es decir, situaciones que añaden vulnerabilidad a la que ya supone la discriminación como mujer o niña, haciéndola más patente en unos casos ("las de abajo") que en otros ("las de arriba") y en unos países (periféricos) que en otros (centrales) $^{16}$.

En España y concretamente en Melilla, en los primeros años de este siglo venimos siendo testigos de la llegada de chicas menores que proceden mayormente de Marruecos, aunque se acreditan casos de posibles menores de origen subsahariano que tras ser valoradas por el forense se determina que ya han cumplido la mayoría de edad.

De Enero a Julio de este año 2011, esta tendencia se mantiene, quedando a cero reducida la incidencia de las menores extranjeras no acompañadas de origen subsahariano ${ }^{17}$. Justamente por las diferencias entre unos (MENAS masculinos) y otras (MENAS femeninas) se hace necesario redactar y fundamentar un trabajo de investigación, cimentado al mismo tiempo en la ausencia de estudios contrastados acerca de esta evidencia ya que son escasas las publicaciones, memorias o tesis realizadas sobre niñas y adolescentes menores no acompañadas frente a la proliferación de tratados sobre los menores en su misma situación. Esta circunstancia ha dado lugar a una errónea creencia acerca de la inexistencia de este nuevo fenómeno social.

Al mismo tiempo, este trabajo se ha de basar en la novedad de lo mostrado y se espera dar a conocer una realidad concreta y diaria: el trabajo con menores extranjeras no acompañadas en Melilla. Esta realidad, en ocasiones pasa desapercibida por la necesidad de inmediatez en las actuaciones tras ser interceptadas (ingreso en centro, tutela...). La urgencia mencionada, ocasiona que no se reflexione acerca de lo que está sucediendo en los entornos sociales y en las familias de estas menores. Se pretende por tanto, ofrecer

creciente demanda para cubrir tareas que se corresponden a la esfera de la reproducción en las sociedades receptoras. Los flujos migratorios hacia los países desarrollados, se han feminizado, es decir, progresivamente son más las mujeres que se desplazan de forma autónoma e independiente, no ya para reagruparse con su pareja o familia, sino para iniciar un proyecto de vida, insertándose en el mundo laboral de la sociedad de acogida, intentando cumplir con las expectativas de futuro que ellas mismas diseñan, en Carlota Solé y Lorenzo Cachón, "Globalización e inmigración: Los debates actuales”. Revista Reis n 116. Madrid, 2006, pp.13-52.

${ }^{16}$ José María Tortosa, "Feminización de la pobreza y perspectiva de género", en Revista Internacional de Organizaciones, RIO n 3. Madrid 2009.://www.revista-rio.org/index.php/revista rio/article/view/33

${ }^{17}$ Fuente: Dirección General del Menor y la Familia de la Consejería de Bienestar Social y Sanidad. Ciudad Autónoma de Melilla. 
una visión actualizada de este hecho social (ya que ha sido redactada durante todo el año 2011) y localizada en la ciudad de Melilla desde el año 2006 a nuestros días.

El trabajo está estructurado en diez capítulos con cuatro anexos, en los que se analizan las siguientes cuestiones:

Capítulo I: INTRODUCCIÓN. Se inicia el trabajo presentando una introducción y detallando el Estado de la Cuestión de las MENAS en Melilla, caracterizando al grupo de manera genérica e incluyendo la definición del Derecho Comunitario. Se incluye asimismo la estructura.

Capítulo II: OBJETIVOS. En este capítulo se desarrollan los objetivos tanto generales como específicos. Los objetivos principales han sido determinar el país o países de los que provienen las menores extranjeras no acompañadas en Melilla durante los años 2006-2011 y evidenciar que el fenómeno que nos ocupa se fundamenta en una nueva forma de inmigración infantil.

Los objetivos específicos han sido analizar los procesos de entrada, protección, integración y / o repatriación, o declaración de mayoría de edad de las menores extranjeras no acompañadas en Melilla y por ende en España ofreciendo una visión lo más ajustada posible a la realidad de este colectivo de menores, chicas, en riesgo de exclusión social así como conocer con mayor detalle la situación actual de las menores extranjeras no acompañadas.

Con los objetivos planteados, nos hemos propuesto realizar un estudio en profundidad sobre una realidad apenas estudiada de manera científica para dotar tanto a la Ciudad Autónoma de Melilla como a los ciudadanos de elementos de juicio suficientes acerca de la situación actual de estas menores en la ciudad, así como la pertinencia o no de mayores recursos que atiendan las necesidades observadas o manifestadas por las menores.

Capítulo III: MARCOTEÓRICO. Desplegamos aquí el Marco Teórico con el que hemos operado, destacando los estudios sobre pobreza evidenciada por las menores en su origen, los tratados acerca de la desigualdad social en que se encuentran una vez asentadas en Melilla, los trabajos sobre sociología de la pobreza y las publicaciones sobre los actuales procesos de exclusión social vinculándolos a los presentes procesos migratorios internacionales. 
Uno de los problemas de fondo de nuestras sociedades actuales - no sólo las occidentales- es que las desigualdades y brechas sociales se están ensanchando y que buena parte de la juventud está padeciendo procesos de exclusión social. Una exclusión social que presenta diferentes facetas (laboral, económica, residencial, política, cultural...). En algunos países a todo esto se añaden dimensiones abiertamente políticas (falta de democracia, de derechos y libertades) y situaciones de corrupción y de "monarquización familista" y apropiadora del poder y los privilegios ${ }^{18}$.

Capítulo IV: HIPÓTESIS DE INTERVENCIÓN. Con el presente estudio se han observado múltiples diferencias entre las y los menores extranjeros no acompañados. Las primeras discrepancias se manifiestan como se ha dicho en cuanto al sexo, pero aumentan en cuanto a las relaciones de poder entre géneros ya que éstas jóvenes en sus sociedades de origen, no disfrutan de condiciones de igualdad y por tanto de oportunidad de organizar su vida de manera independiente en ellas se basan nuestras hipótesis de trabajo.

\section{CapítuloV: ESTADO DE LA CUESTIÓN. ACTUALIZACIÓN DE DATOS. Se} concreta con la actualización de datos oficiales facilitados por la Ciudad Autónoma y de las entrevistas realizadas a la Directora General del Menor y la Familia de la Consejería de Bienestar Social y Sanidad de la Ciudad Autónoma de Melilla; Jefe de Policía Local Grupo de Menores; Jefe de Policía Nacional Grupo de Extranjería y Teniente Coronel de la Guardia Civil acerca de la situación actual de las menores extranjeras no acompañadas en Melilla.

Entre los años 1993 y 2009 se observa un aumento del número de acogidas sobre todo a partir del año 1994 (tal vez porque no se dispongan de datos) advirtiéndose un leve descenso en el año 1997. Se mantiene la tónica ascendente hasta el año 2004, siendo 1998 el año del inicio del ascenso que se conoce hoy. A partir de este año el aumento se mantiene con cifras máximas en 2002 y 2003, volviendo a caer en el año 2004 cifra que

${ }^{18}$ José Félix Tezanos, Juan José Villalón y Verónica Díaz, Tendencias de cambio de las identidades y valores de los jóvenes en España. 1995-2007, INJUVE, Madrid, 2008; José Félix Tezanos, Juan JoséVillalón y Verónica Díaz Moreno, La juventud hoy: entre la exclusión y la acción. Tendencias de identidades, valores y exclusión social de las personas jóvenes, INJUVE, Madrid. 2009; José Félix Tezanos (ed.), “Juventud y exclusión social”, Editorial Sistema, Madrid, 2009; José Félix Tezanos, Juan José Villalón, Verónica Díaz y Vania Bravo, El horizonte social y político de la juventud española, INJUVE-Fundación Sistema, Madrid, 2010. 
con mucha probabilidad no se corresponde con la real ${ }^{19}$. En el año 2005 el número de menores vuelve a aumentar y se pueden comprobar los niveles similares junto con los años 2006 y 2007. El año 2008 es el que ofrece un mayor crecimiento de la entrada de MENAS en España, aunque el fenómeno reduce en $2009^{20}$.

\section{GRÁFICO I. \\ EVOLUCIÓN DE LOS MENORES EXTRANJEROS INMIGRANTES NO ACOMPAÑADOS ACOGIDOS POR ESPAÑA (AÑOS 1993-2009)}

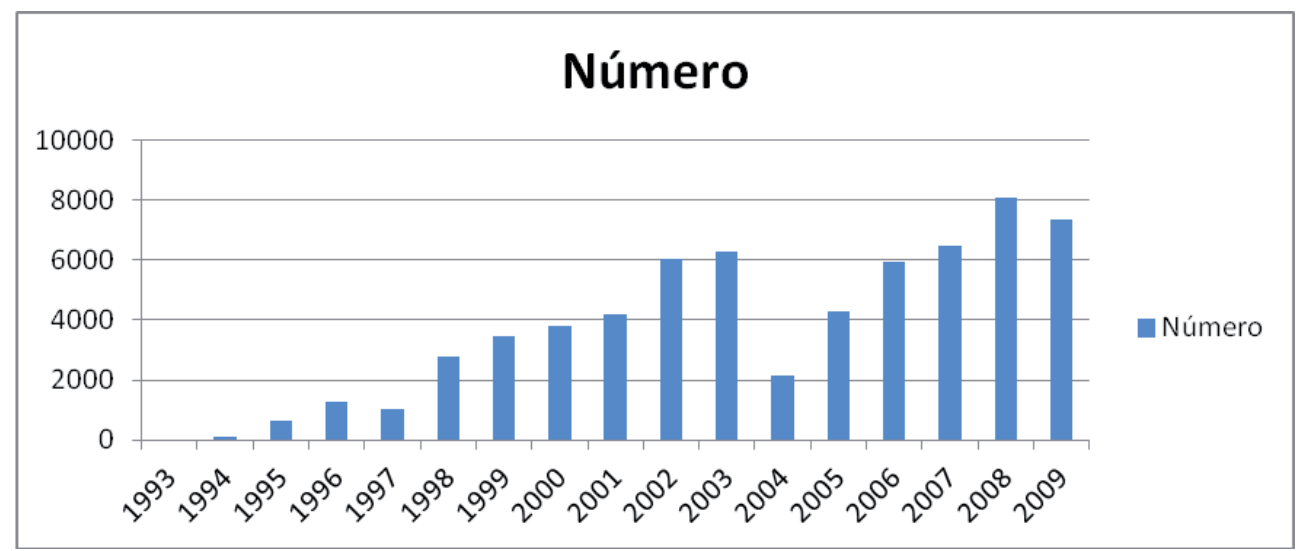

Fuente: del año 1993 al 1999: Diputación Foral de Araba, Diputación Foral de Bizkaia, Informes sobre "La situación de los menores extranjeros no acompañados" remitidos por las CCAA al Ministerio de Trabajo y Asuntos Sociales por el Grupo de Trabajo reunido el 29 de febrero del 2000, Junta de Castilla y León, Generalitat Valenciana y Gobierno de Aragón. Del 2000 al 2009: Ciudad Autónoma de Melilla, Diputación Foral de Araba, Diputación Foral de Bizkaia, Diputación Foral de Gipuzkoa, Generalitat de Catalunya, GeneralitatValenciana, Gobierno de Aragón, Junta de Castilla y León, Junta de Comunidades de Castilla-La Mancha, Junta de Andalucía, Ministerio de Interior, Ministerio de Trabajo e Inmigración, Región de Murcia, Xunta de Galicia y Vaciado de expedientes de los Centros de Primera Acogida de la Comunitat Valenciana del equipo investigador del proyecto en esta CCAA, 2000 y 2007/08/10.

${ }^{19}$ En el año 2004 la competencia de la recopilación de datos sobre los y las MMNA pasó del Ministerio del Interior al Ministerio de Trabajo y Asuntos Sociales, con lo cual CCAA y 2 ciudades autonómicas no facilitaron los datos de ese año (Canarias, Madrid, Ceuta, Melilla, Castilla-la Mancha, Cantabria, Extremadura, Baleares, Asturias, La Rioja y Navarra) y tampoco del año 2005. De estas, hay 2 CCAA y las 2 ciudades autonómicas que son de vital importancia en el recuento final de menores detectados en años anteriores.

${ }^{20}$ Estos datos, en general, deben tomarse como aproximativos, ya que desde el Ministerio se afirma que hay muchas dificultades para recogerlos bajo los mismos criterios en cada caso. 
Capítulo VI: METODOLOGÍA. Detallamos la Metodología empleada. Exponemos cómo se ha realizado el estudio, los pasos cursados y las técnicas de investigación aplicadas.

El estudio se ha centrado en el análisis de tipo diacrónico de los expedientes de protección incoados a menores en situación de protección catalogadas como MENAS desde los Servicios Sociales en cinco años, desde Julio de 2006 a Julio de 2011. La investigación se ha llevado a cabo con la información facilitada por las Administraciones y las menores. Ellas han relatado de primera mano qué opinan de su propio proceso migratorio y de las consecuencias de su decisión, así como de las perspectivas de inicio y las actuales.

Se han realizado un total de 32 entrevistas en profundidad. Además de las efectuadas a las menores, se han mantenido otras con diversos profesionales que trabajan con este colectivo. Se han plasmado:

- 11 entrevistas con las MENAS tuteladas autorizadas por la Consejería de Bienestar Social y Sanidad vitales y 1 con una exMena (mayor de edad). En ellas se han abordado las opiniones de las menores sobre su propia realidad y sus experiencias, profundizando en la situación personal y familiar que tenían en sus países de origen, en las motivaciones que tuvieron para emigrar y sobre quién tomó la decisión de iniciar esta aventura. También se ha ahondado en el grado de cumplimiento de sus expectativas, preguntándoles en última instancia si volverían a repetir esta aventura migratoria. Señalar que todas tienen nacionalidad marroquí ya que no se ha podido acreditar el ingreso de ninguna menor subsahariana durante el presente estudio (las supuestas menores de origen subsahariano que llegan a los centros resultan no serlo una vez comprobada su edad por el Equipo de Forenses).

Capítulo VII: PERFILES. Se exponen los Perfiles de las Menores, señalando y estableciendo diferencias entre ellas necesarias para estudiar su situación familiar en origen, proyectos migratorios, expectativas además de la valoración de su situación actual y de futuro.

Capítulo VIII: TENDENCIAS Y RETOS DE FUTURO. Como corolario se exponen las tendencias de futuro evidenciadas y la realidad actual que viven las menores en nuestra ciudad ya que la investigación se ha desarrollado con la intención de ser descriptiva, explicativa y predictiva. El trabajo de campo se ha realizado durante los meses de Enero a Julio de este año 2011. 
Se describen las tendencias y los retos de futuro previstos tras los análisis efectuados sobre esta realidad desde un doble plano: el fenómeno en su conjunto desde el punto de vista de los profesionales y las expectativas de las menores de manera particular.

Capítulo IX: CONCLUSIONES. Esta investigación finaliza con las principales conclusiones obtenidas.

Capítulo X: BIBLIOGRAFÍA Y ANEXOS. Pormenorizamos tanto las fuentes bibliográficas y normativas (Directivas, Informes, Reglamentos y Resoluciones), así como las páginas web y de prensa de las que se puede obtener información. Por último se señala la relación de entrevistas y su texto original.

\section{COROLARIO:}

Como ya se ha dicho, el presente artículo pretende ser un resumen delTrabajo Fin de Máster, previo al Doctorado en "Análisis de los Problemas Sociales”. Pero: ¿Porqué elegir como tema de investigación las "MENAS" en Melilla?

1.- Por ser un fenómeno nuevo, datado en España a partir de finales de los años 80.

2.- Por revelar profusas diferencias con el fenómeno protagonizado por los chicos.

3.- Por no existir apenas estudios contrastados sobre este fenómeno.

4.- Por ser un hecho social invisibilizado.

\section{BIBLIOGRAFÍA:}

- (Directivas 2003/9/CE del Consejo, de 27 de enero de 2003 y la Directiva 2001/55/ CE, del Consejo, de 20 de julio de 2001).

- Bernabé López García, "La inmigración de magrebíes y africanos. Asumir la vecindad". Alicia Alted y Almudena Asenjo (Coord.). De la España que emigra a la España que acoge. Caja Duero-Fundación Largo Caballero, Madrid, 2006, pp. 480-491.

- Carlota Solé y Lorenzo Cachón, "Globalización e inmigración: Los debates actuales". Revista Reis $\mathrm{n}^{\circ}$ 116. Madrid, 2006, pp.13-52.

- http://medios.mugak.eu/noticias/noticia/96151

- http://www.dianova.es/content/que-hacemos-menas-residencial.html http://www.revista-rio.org/index.php/revista rio/article/view/33 
- http://www.ujaen.es/investiga/ideo/jornadas/recursos/actasConISBN.pdf http:/ /menoressolos.blogspot.com/2009/06/las-ninas-y-adolescentes-queemigran.html

- Informe producido por la Red Europea de Migraciones, elaborado por el Punto de Contacto Nacional de España "La política de acogida, repatriación y acuerdos para la integración de los Menores extranjeros no acompañados”. Madrid. Junio 2009.

- José María Tortosa, "Feminización de la pobreza y perspectiva de género", enRevista Internacional de Organizaciones, RIO nº 3. Madrid 2009.

- Manuel Jiménez Álvarez. Los niños de la calle y el fenómeno de la emigración de menores: análisis de los contextos migratorios en el área urbana de Tánger. Agencia Española de Cooperación, Madrid, 2001.

- Prolegómenos: Derechos y Valores. "Menores extranjeros no acompañados. La situación en España”. Universidad Militar Nueva Granada. Bogotá. Colombia. Enero/junio 2007, pp.149-162.

- Reglamento aprobado por el Real Decreto 2393/2004, de 30 de diciembre, que desarrolla Ley Orgánica 4/2000, de 11 de enero, sobre derechos y libertades de los extranjeros en España y su integración social, modificada por las Leyes Orgánicas 8/2000, de 22 de diciembre, 11/2003, de 29 de septiembre y 4/ 2003, de 20 de noviembre.

- Resolución de 24 de marzo de 2009 (BOE de 2-4-2009). Fondos de Apoyo a la acogida y la integración de inmigrantes así como el refuerzo educativo de los mismos.

- Secretaría de Estado de Inmigración y emigración. http://extranjeros.mtin.es/es/ RedEuropeaMigraciones.

-Violeta Quiroga y Montserrat Sòria, "Los y las menores migrantes no acompañados/as: entre la indiferencia y la invisibilidad”, en Revista de Educación Social, $n^{\circ} 45$. Madrid, 2010, p13 p35. 